\title{
Earth fault detection in distributed power systems on the basis of artificial neural networks approach
}

DOI:10.36909/jer.13627

\begin{abstract}
Ali Ahmadi, Ebrahim Aghajari*, Mehdi Zangeneh
Department of Electrical Engineering, Ahvaz Branch, Islamic Azad University, Ahvaz, Iran.

*Email: aghajari@iauahvaz.ac.ir; Corresponding Author.
\end{abstract}

\begin{abstract}
Nowadays, the advancement of microgrids promises numerous economic and environmental advantages of renewable energies to nations and societies. The presence of decentralized energy units, however, makes serious technical challenges; for instance, criteria and procedure of fault recognition and diagnosis in this condition is entirely changing. This article, therefore, proposed a novel accurate and fast technique based on Artificial Neural Networks (ANN) for earth fault detection. A sample distributed power system considered for the proposed technique and different earth faults applied to this system consist of one phase, two phases and three phases faults. Also, any alteration of current and voltage signals of all phases is investigated at the fault occurrence moment. Analysis of simulation results demonstrates how the proposed technique could make faster responses and improve the reliability of the distributed power system by more accurate fault recognition in comparison with the other traditional methods such as the Wavelet Transformation technique. The proposed technique is likely to enhance the growth of renewable energy sources usage by decreasing operational risk factors and fault recognition delays.
\end{abstract}

Key words: Artificial Neural Networks (ANN), Distributed energy system, Earth fault detection. 


\section{INTRODUCTION}

The appropriate performance of the Decentralized Generation (DG) has become increasingly prominent due to the increasing environmental pollution caused by consumption of fossil fuels and development of renewable energy technologies over the past years. The performance conditions of the distributed system are changed while there are DG units in the system. These changes, especially, are most significant in protection criteria [1-4].

There are a variety of faults in electrical power distribution systems, however, this paper focuses on earth fault detection. This class of faults is one of the usual events in mediumvoltage distribution networks. But, due to the moderate current and high impedance of the path, the conventional protection relays don't respond appropriately to these types of faults [5-8].

In the past years, several techniques have been reported with the purpose of fault recognition and classification. In all methods, extracting the characteristics of the fault signal is essential (Dehghani, Khooban, and Niknam 2016). These methods consist of expert systems and fuzzy logic (Nan, Khan, and Iqbal 2007; Veljko, Predrag, and Zeljko 2010) Petri nets (Jiang et al. 2017; Yang 2003), Bayesian networks (Brandt, Jervis, and Maidon 1997; Yongli, Limin, and Jinling 2006), , granular computing (Xiuping Xu and Peters 2002) and rough sets (Rawat et al. 2014). Also (Xin-min et al. 2007) investigates Realvalued Negative Clone Selection on the basis of Higher-order Statistics approach. Reference (He and Blum 2011) proposed the Hypothesis Testing method. Furthermore, [20-22] considers the Vector Surge technique while (Wester 1998) studies different mechanical and electrical techniques for earth fault detection. Reference (Ndou et al. 2013) has suggested Under/Over Voltage relays, as well. Another important method for fault detection in distributed systems is the Wavelet Transformation approach, which has interested several researchers in this area. This method first was proposed for seismic data analysis (Grossmann and Morlet 1984) and then it used to relay distributed power systems (Chaari, Meunier, and Brouaye 1996). Therefore, (Samui 
and Samantaray 2012) proposed the Wavelet Singular Entropy technique for islanding recognition. Reference (X Xu and Kezunovic 2002) studies fault characteristic extraction through Wavelet Transformation technique and (Escudero et al. 2017) presents a method on the basis of Wavelet and Park's Vector Transformation (WPVT) approach.

However, the major difficulty in the Wavelet Transformation method is selecting a proper threshold value. If it is set on a small value, some particular transient fault-like situations of the system (such as capacitor switching and inrush current of transformers) may be identified as a fault. On the contrary, if a large value is opted for it, because of the high impedance of earth fault, it could not be recognized by the protection system. Therefore, (Chen et al. 2014) tries to create a new accurate criterion through experimental trials. This problem, however, could be effectively vanished with the proposed technique in this study.

Typically, fault protection as one of the fundamental parts of transmission line systems is encountered certain challenges as follows: by taking into account nonlinearity and timevarying of earth faults, the most crucial problem is accurate recognition of faults and transient fault-like situations. The response speed obviously is the other vital factor that directly depends on the detection method and its sampling rate. Moreover, the definition of a new fault type for traditional protection systems is too expensive because it needs relays modification or replacement. These problems have been made earth fault detection extremely challenging.

Therefore, this article has developed an ANN based technique to tackle all mentioned difficulties. Accordingly, the main contributions of this paper consist of: (1) The proposed protection method could successfully recognize symmetric and unsymmetric earth faults and separates them from the normal transient conditions of the power system. (2) Trainability, reducing sampling rate and powerful theoretical support of the suggested ANN method has made implementation and performance of the protection system notably fast, cost-efficient and simple. Eventually, this capable technique makes microgrids more reliable and safer by 
making a fast response and preventing expansion of fault over the grid.

The rest of this paper is organized as follows. Section two provides a brief explanation of the ANN method. Section three presents the distributed system and its criteria considered in this study for simulation purposes. Section four analyzes and discusses the simulation results. Section five, finally, provides conclusions and future scope.

\section{DISTRIBUTED POWER SYSTEM}

This paper considers the distributed system described in (Hasheminejad et al. 2016) that includes four generators with power supply capacities of 1000MVA, one step down transformer to reduce the voltage from $765 \mathrm{Kv}$ to $500 \mathrm{Kv}$ and five $500 \mathrm{Kv}$ buses. Figure 1 shows the one-line diagram of this system and the length of transmission lines between buses as well. Moreover, the system's frequency is selected $50 \mathrm{HZ}$.

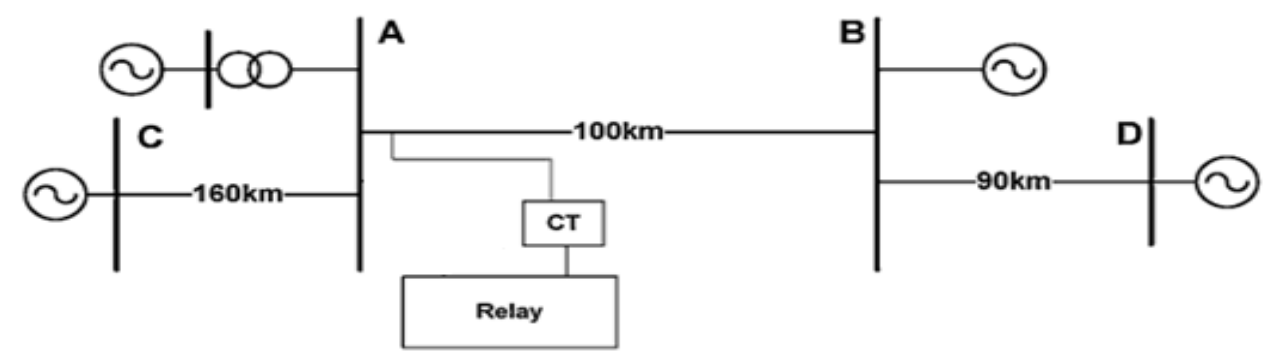

Figure 1 One-line diagram of the distributed system (Hasheminejad et al. 2016)

The transmission line across bus A to B is considered as fault protected line; the protection system, moreover, is placed at bus A that named Relay in Figure 1. The line's current information, furthermore, is measured by a current step down transformer of 1600A/5A that has 1 Tesla magnetic flux density at its knee point for every phase. It's considered that all faults occur at $50 \mathrm{Km}$ distance from bus $\mathrm{A}$ as well. It's noteworthy that fault detection in this system is performed by the current analysis of three phases. Table 1 shows the transmission lines properties of the distributed system. 
Table 1 Properties of transmission lines

\begin{tabular}{|c|c|c|}
\hline Row & Parameter & Value \\
\hline 1 & Per unit impedance (zero sequence) & $0.2638 \mathrm{Ohm} / \mathrm{Km}$ \\
\hline 2 & Per unit impedance (positive sequence) & $0.0195 \mathrm{Ohm} / \mathrm{Km}$ \\
\hline 3 & Per unit inductance (zero sequence) & $0.0359 \mathrm{H} / \mathrm{Km}$ \\
\hline 4 & Per unit inductance (positive sequence) & $0.0106 \mathrm{H} / \mathrm{Km}$ \\
\hline 5 & Per unit capacitance per unit (zero sequence) & $5.75 \mathrm{e}-9 \mathrm{~F} / \mathrm{Km}$ \\
\hline 6 & Per unit capacitance (positive sequence) & $8.74 \mathrm{e}-9 \mathrm{~F} / \mathrm{Km}$ \\
\hline
\end{tabular}

The specified distributed system is created by SimPowerSystems toolbox in MATLAB (version R2018b). For simulation purposes, a desktop computer is used with an Intel Core i7 processor, $6 \mathrm{~GB}$ of RAM memory and Windows 7 operating system. The distributed power system, additionally, is illustrated in Figure 2.

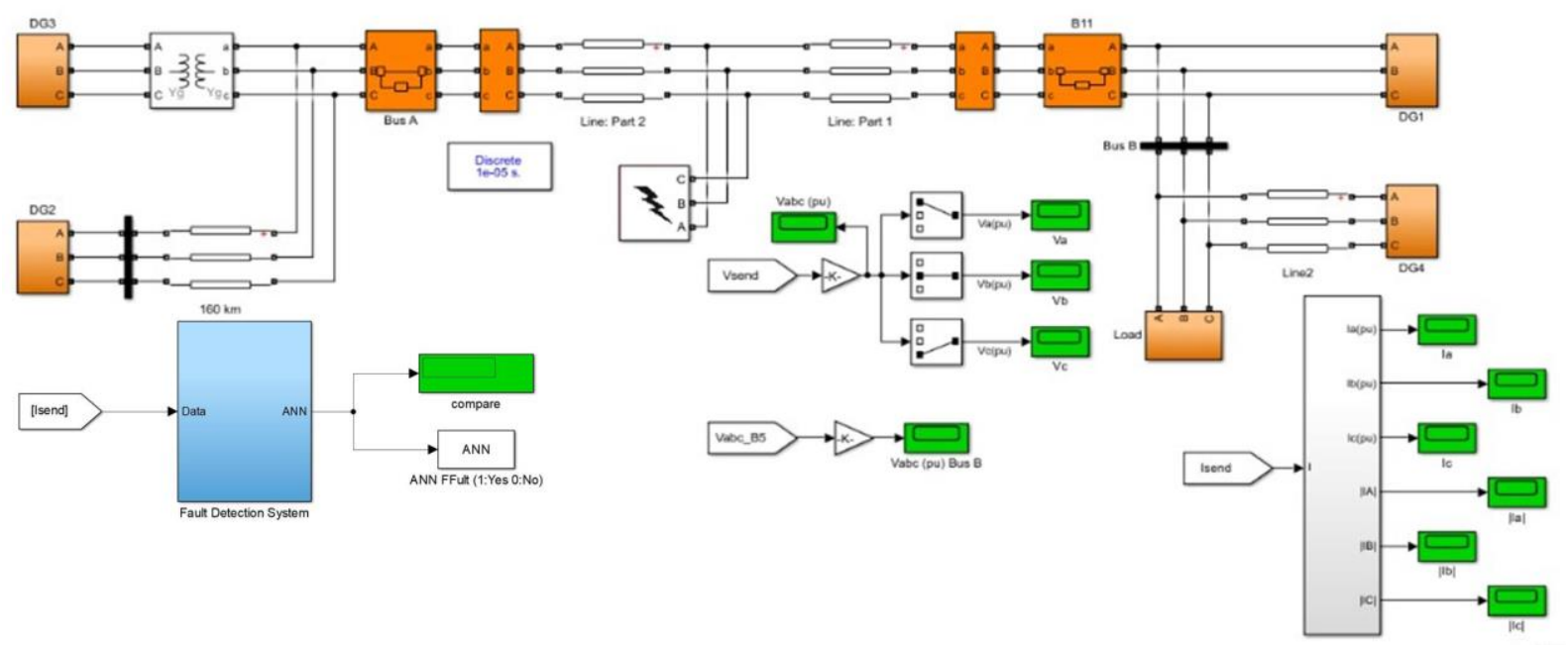

Figure 2 Simulation of the electric distribution system

\section{ARTIFICIAL NEURAL NETWORKS}

ANN method as one of the sub-branches of artificial intelligence has two crucial abilities include training and predicting. This approach was inspired by the biological performance of the human brain that considers a collection of inputs as a training dataset and behaves based on it (Ozkan \& Erdemir, 2021; Ulas \& Ozkan, 2019). Every ANN model includes a set of source nodes (units) as the input layer, a collection of hidden nodes that make at least one 
hidden layer, and one output layer as well. The number of units in the input-output layers only depends on the number of variables in input and output. But, the number of hidden layers and their nodes affects the generalization capability of the neural network (Özkan et al., 2021; Ozkan \& Erdemir, 2020).

In an ANN model, the hidden layer has a vital role hence it must be organized carefully, if a small value opted for the layers and its nodes, the performance of the network may not be satisfactory and if it is selected too large, it will be over-fitting and the network performs a poor generalization on the new data. Moreover, the training phase of a MLP means determining parameters of the network include weights of connections. In fact, it needs to find the best value for the weights in the way that the error between the actual output and the similar value of training data will be minimal (Ulas et al., 2019).

In this study, Levenberg-Marquardt algorithm and Multilayer Perceptrons (MLPs) class has been used for modeling the ANN approach. The training algorithm is the mean square error and the applied activation functions are piece-wise linear, hyperbolic tangent and logistic function, as shown in Eqs.(3)-(5), respectively.

$$
\begin{gathered}
\varphi(v)=v \\
\varphi(v)=\frac{1-e^{-2 v}}{1+e^{-v}}=\frac{2}{1+e^{-v}}-1 \\
\varphi(v)=\frac{1}{1+e^{-v}}
\end{gathered}
$$

Considering that the ANN model has been developed to detect earth faults throughout the electricity transmission lines then the input layer has nine nodes that include sensed data of the three continuous samples of each three phases. The output aimed to determine fault occurrence therefore it only has one node in that, the range between $\left[\begin{array}{ll}0 & 0.5\end{array}\right]$ refers to the normal condition of the distributed system and the range [ $\left[\begin{array}{ll}0.5 & 1\end{array}\right]$ demonstrates fault occurrence. Although different numbers have been considered for the hidden layer nodes, the 
satisfactory response has been produced by ten units. Figure 3 shows the feedforward developed ANN model.

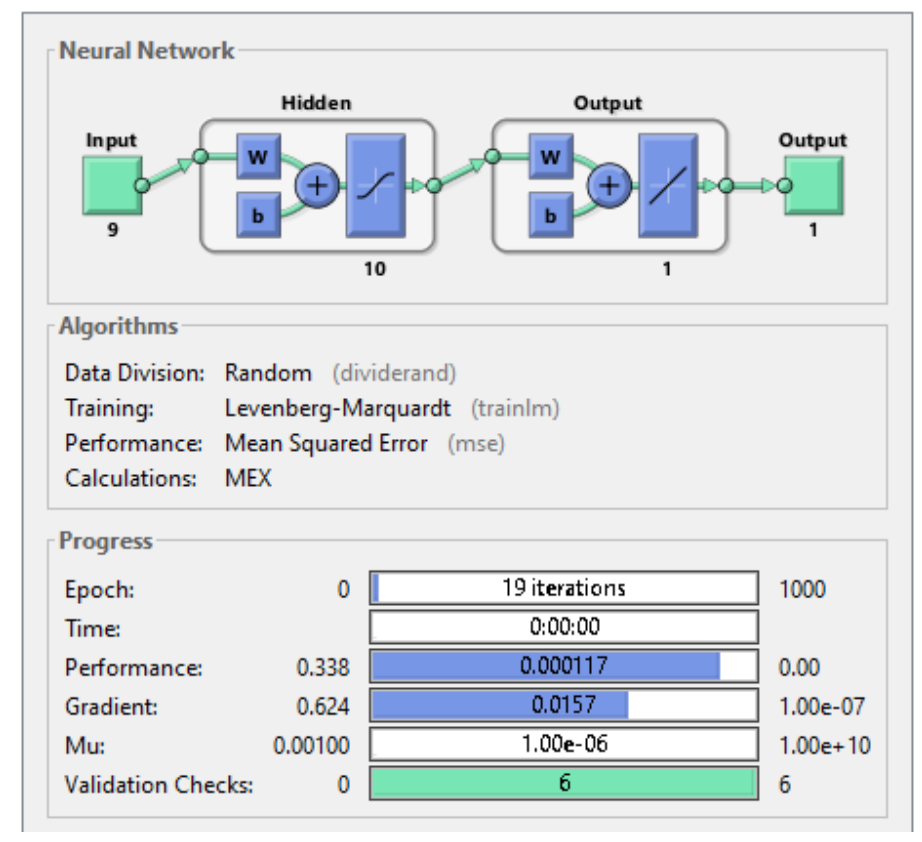

Figure 3 Training process of feedforward ANN model

Moreover, the ANN method needs a suitable training dataset to respond accurately to the fault circumstances. This required data is obtained by running the simulation model in the last section for many operating modes include normal and fault conditions. As could be seen in Figure 3, the training process has been stoped in the 19th iterations. Training error diagram in terms of repetition is shown in Figure 4 in which the best validation performance has been marked.

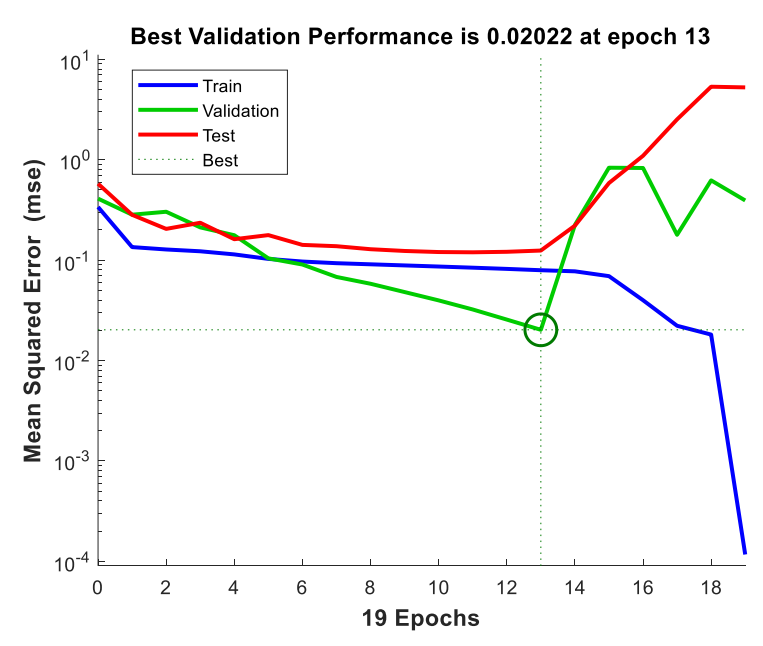

Figure 4 The ANN model best training validation 
The error histogram, train process, validation and test of the developed ANN model, are illustrated in Figure 5 and 6 respectively. These diagrams have been obtained by MATLAB software using ANN toolbox and appropriate code.

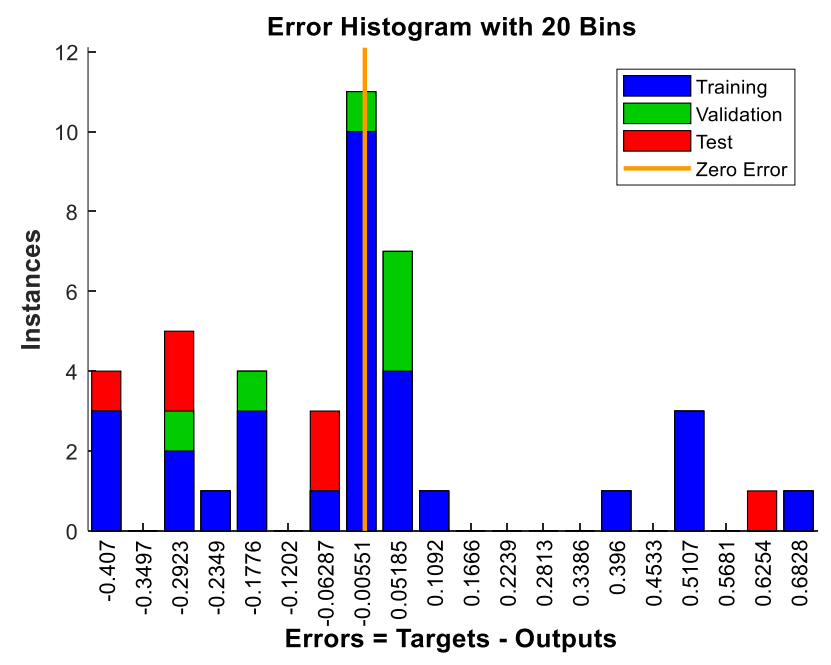

Figure 5 The ANN model error histogram
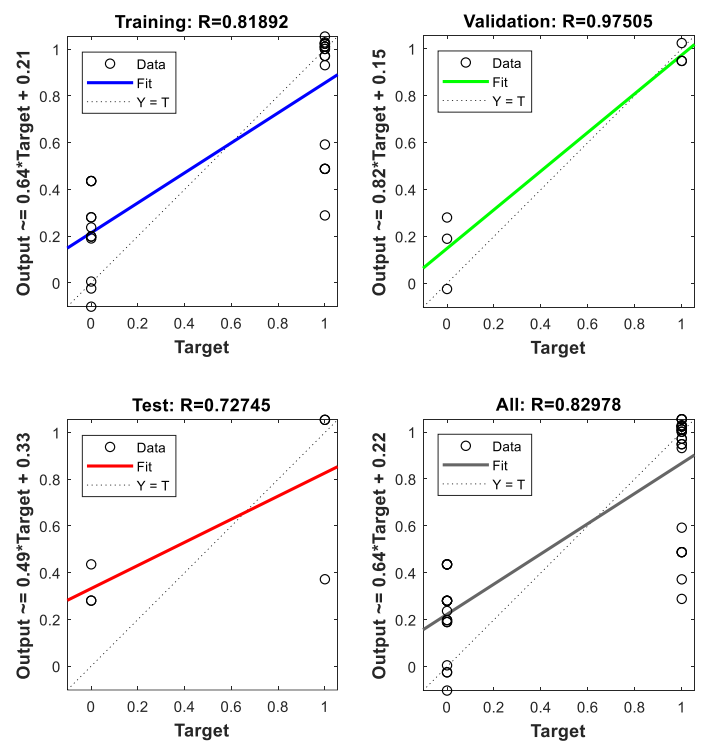

Figure 6 The ANN model training, validation and test

\section{SIMULATION RESULTS}

In this section, the performance of the proposed method is investigated by applying different types of fault to the specified distributed system. These faults include symmetrical and 
unsymmetrical earth faults described in (Grainger, Stevenson, and Stevenson 2003). A competitive analysis, moreover, has been performed between the proposed method performance and the WPVT technique presented in (Escudero et al. 2017). The considered faults are as follows:

- Symmetrical three-phase to the earth

- Unsymmetrical two-phase to the earth

- Unsymmetrical one-phase to the earth

Also, the sampling frequency of $100 \mathrm{kHz}$ is selected for all simulations and it is assumed all faults occurred at 0.06 seconds.

The first investigated fault case is a symmetrical three phase to the earth in the transmission line that connects bus A to B. Also, it is assumed that the fault resistance is $20 \mathrm{ohms}$. Due to this condition, the current and voltage's waveform of the line will fluctuate. Figure 7 and 8 illustrates these fluctuations in this circumstance.

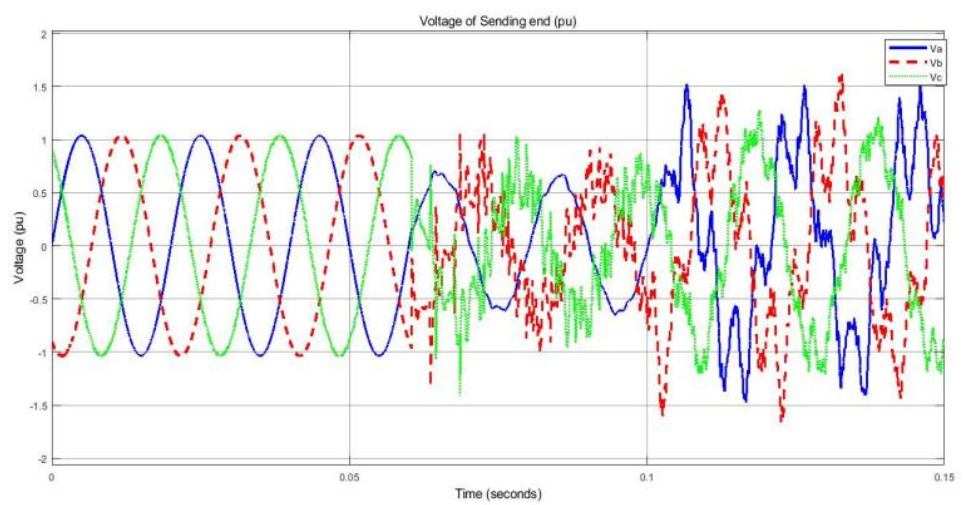

Figure 7 Voltage variations in the case of symmetrical three-phase to the earth fault

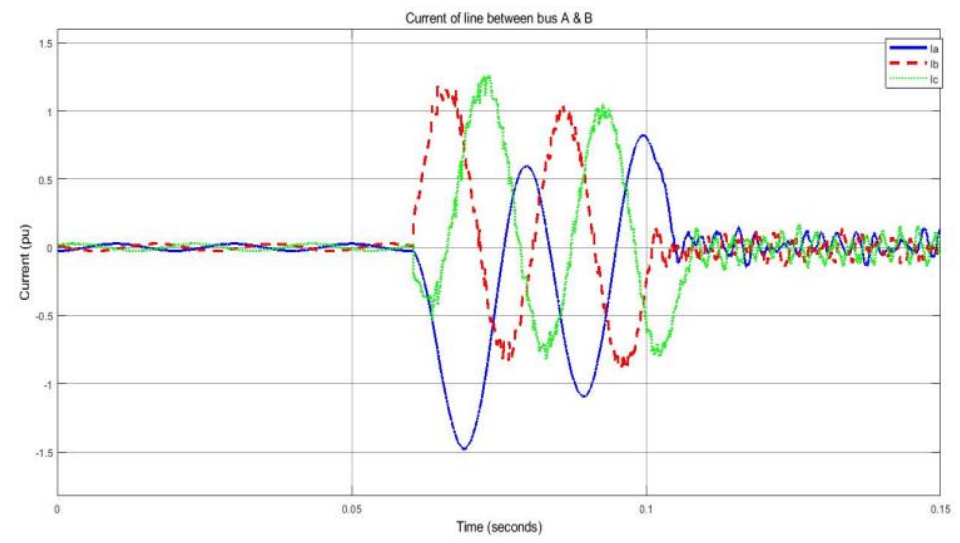

Figure 8 Current variations in the case of symmetrical three-phase to the earth fault 
Based on the method presented in (Escudero et al. 2017), initially, the park parameters are extracted from the current signal of the line in which fault occurred. Then, the Haar Wavelet transformation is applied to these parameters, and approximation diagrams are obtained.

For the purpose of recognizing this type of fault by the WPVT technique, the threshold limit value needs to be selected very low (in such a case 0.05 ). Nonetheless, it is impossible to select such a small value in practice since thereby it could be the usual transient normal conditions of the distributed system are also recognized as a fault. The fault, however, detected by the WPVT and ANN methods at 0.1028 and 0.063 seconds respectively.

In the first unsymmetrical fault case, it is assumed that two phases are connected and then it comes into contact with the earth. In addition, the impedance of the earth is assumed 1000 ohms. As a result of this situation, the current and voltage waveform of the phases changed, as illustrated in Figure 9 and 10.

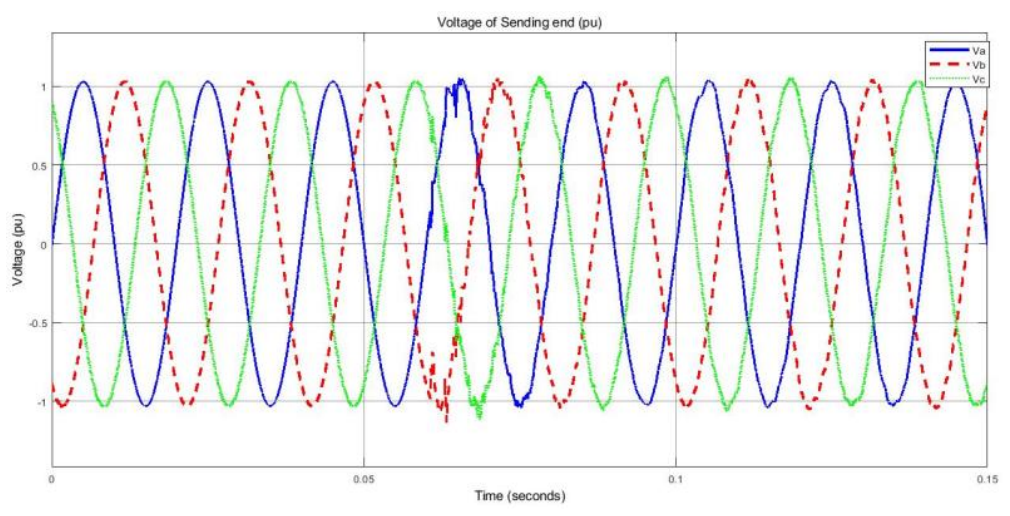

Figure 9 Voltage variations in the case of unsymmetrical two-phase to the earth fault

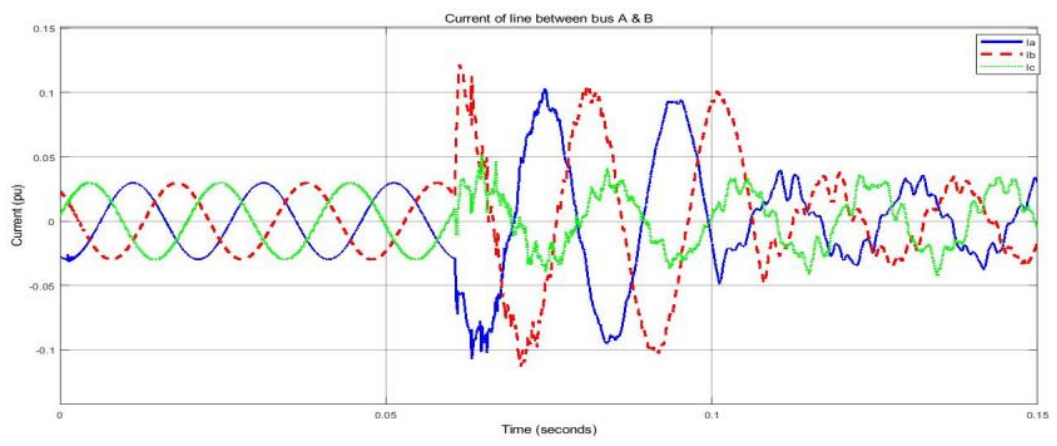

Figure 10 Current variations in the case of unsymmetrical two-phase to the earth fault 
As can be observed, in this situation the waveform of voltage is slightly changed because the impedance of the earth fault is too high. Also, the current waveform changes are not dissimilar with the usual grid states. In such a condition fault recognition is difficult particularly in methods that compare current samples with a constant threshold and former samples.

At the moment of fault, some more frequency components add to the frequency terms of the original signal which makes distinct parameters of the park transform. The WPVT and ANN methods, however, sensed this fault at 0.109 and 0.06174 seconds, respectively.

In the second unsymmetrical fault case, one phase is connected to the earth and the impedance of the earth is assumed as $100 \mathrm{ohms}$. As a result of this fault, the current and voltage waveforms have fluctuated slightly. Figure 11 and 12 illustrates the transmission line waveforms.

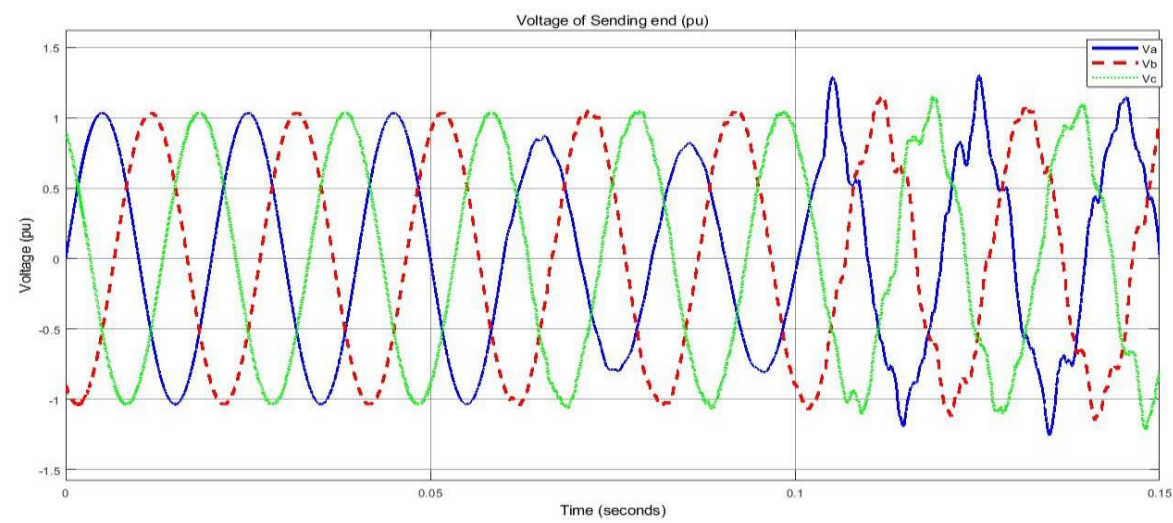

Figure 11 Voltage variations in the case of unsymmetrical one-phase to the earth fault

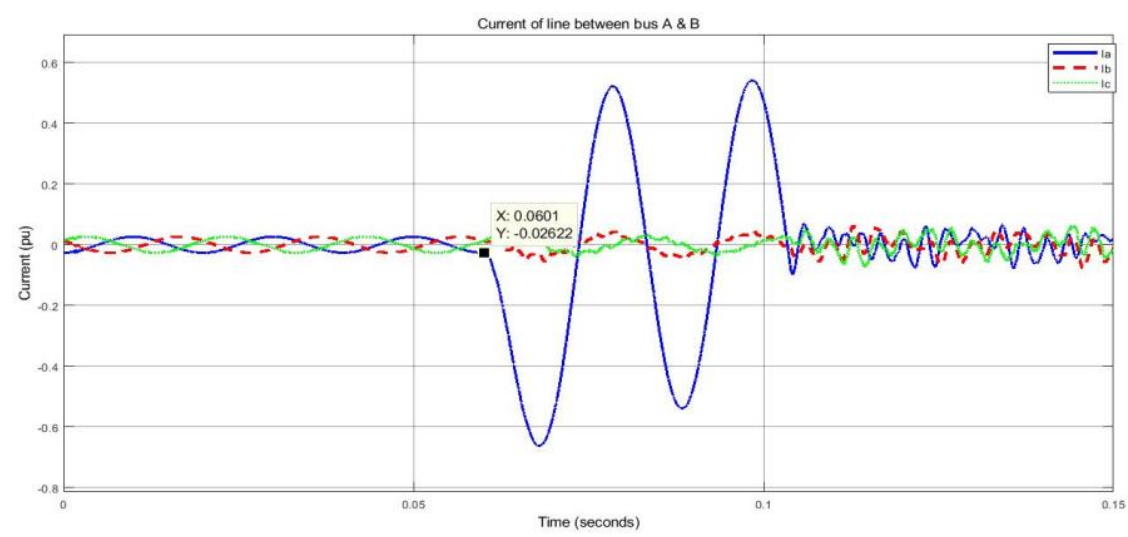


Figure 12 Current variations in the case of unsymmetrical one-phase to the earth fault

In this case, by choosing 0.5 for the threshold limit value, this fault was recognized at 0.079 seconds by the WPVT method. Also, the ANN method detected this fault at 0.062 seconds.

A detection time comparison between the two methods is illustrated in Figure 13. As mentioned before the faults are occurred at 0.06 seconds and the ANN method is detected all three types of faults in less than 3 milliseconds after it happens. While the WPVT technique is recognized the unsymmetrical one phase fault in 19 milliseconds, also it sensed the other two faults in less than 50 milliseconds. Figure 13 clearly shows the faster response of the ANN method in compassion to the WPVT method.

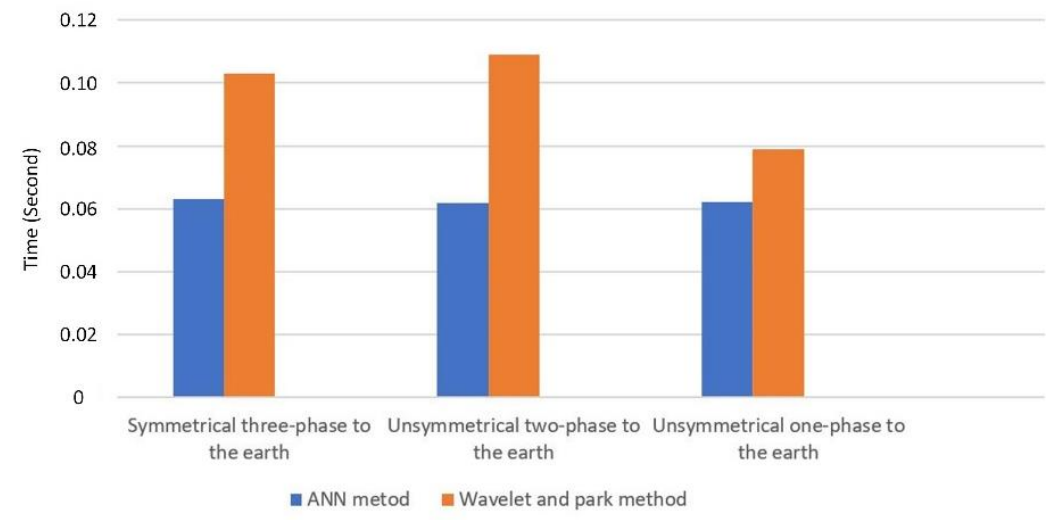

Figure 13 The detection time comparison between WPVT and ANN methods

Another remarkable characteristic of the ANN method is its trainability. With an adequate dataset and appropriate training, the protection system will perform fault recognition quicker and more accurately. Also, it is attainable to have a reliable protection system with a desirable impact of operational risk.

In addition, if new fault mods occur in the distributed system, the new modes can simply be defined as a fault for the ANN protection system and with the appropriate training, it can detect all same mods across the system. Whereas, in traditional fault detection systems new faults are definable with the replacement of all relays through the system which is very 
expensive to implement.

The last comparison between the two methods is based on the number of correct fault detection. As Figure 14 illustrates, 120 different symmetrical and unsymmetrical faults are examined with both methods. The ANN is found 117 faults correctly and the WPVT method is detected 110 cases which means the proposed method improves the reliability of the system up to about $98 \%$.

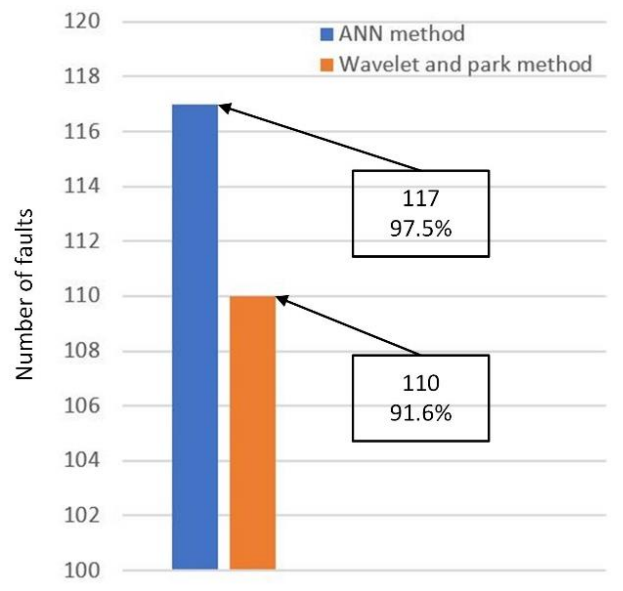

Figure 14 The detection number comparison between WPVT and ANN methods

\section{CONCLUSION}

This article proposed a novel ANN based protection system for microgrids. The effectiveness of this system, moreover, was investigated by the implementation of a distribution network and applying the different earth fault modes in the MATLAB simulation environment. The fault mods examined in this study include one phase, two phases and three phases earth faults which the suggested method successfully recognized all faults and made faster responses in comparison to traditional methods. The results, furthermore, proved that the protection scheme could successfully satisfy all design objectives in particular the accurate detection and quick response. It is expected that this powerful approach reduces the operational risks and extends the reliability of distributed systems; in particular, microgrids that consist of renewable energy resources.

This paper, however, concentrated on grid-connected microgrids although island mode 
situations make different criteria for fault detection. Therefore, these conditions will be analyzed in future studies.

\section{REFERENCES}

Abdali, Ali, Kazem Mazlumi, and Reza Noroozian. 2019. "High-Speed Fault Detection and Location in DC Microgrids Systems Using Multi-Criterion System and Neural Network." Applied Soft Computing 79: 341-53.

Brahma, Sukumar M, and A Adly Girgis. 2004. "Development of Adaptive Protection Scheme for Distribution Systems with High Penetration of Distributed Generation.” IEEE Transactions on power delivery 19(1): 56-63.

Brahma, Sukumar M, and Adly A Girgis. 2002. "Microprocessor-Based Reclosing to Coordinate Fuse and Recloser in a System with High Penetration of Distributed Generation.” In 2002 IEEE Power Engineering Society Winter Meeting. Conference Proceedings (Cat. No. 02CH37309), IEEE, 453-58.

Brandt, Y, B W Jervis, and Y Maidon. 1997. "Circuit Multi-Fault Diagnosis and Prediction Error Estimation Using a Committee of Bayesian Neural Networks.”

Chaari, Oinis, Michel Meunier, and Francoise Brouaye. 1996. "Wavelets: A New Tool for the Resonant Grounded Power Distribution Systems Relaying." IEEE Transactions on Power Delivery 11(3): 1301-8.

Chen, J C et al. 2014. "Detection of High Impedance Faults Using Wavelet Transform.” In 2014 Australasian Universities Power Engineering Conference (AUPEC), IEEE, 1-6.

Chen, J C, B T Phung, T R Blackburn, and D M Zhang. 2013a. "Study on High Impedance Fault Characteristics.” In AUPEC Conf.,.

_. 2013b. "Use of MV Current Transformers as Sensors for High Impedance Fault Detection.” In ISH Conf.,.

Dehghani, Moslem, Mohammad Hassan Khooban, and Taher Niknam. 2016. "Fast Fault Detection and Classification Based on a Combination of Wavelet Singular Entropy 
Theory and Fuzzy Logic in Distribution Lines in the Presence of Distributed

Generations.” International Journal of Electrical Power \& Energy Systems 78: 455-62.

Doyle, Michael T. 2002. "Reviewing the Impacts of Distributed Generation on Distribution System Protection.” In IEEE Power Engineering Society Summer Meeting, IEEE, 103-5.

Escudero, Ricardo, Julien Noel, Jorge Elizondo, and James Kirtley. 2017. "Microgrid Fault Detection Based on Wavelet Transformation and Park’s Vector Approach.” Electric Power Systems Research 152: 401-10.

Freitas, Walmir, and Wilsun Xu. 2004. "False Operation of Vector Surge Relays.” IEEE Transactions on Power Delivery 19(1): 436-38.

Freitas, Walmir, Wilsun Xu, Carolina M Affonso, and Zhenyu Huang. 2005. "Comparative Analysis between ROCOF and Vector Surge Relays for Distributed Generation Applications.” IEEE Transactions on power delivery 20(2): 1315-24.

Freitas, Walmir, Wilsun Xu, Zhenyu Huang, and Jose C M Vieira. 2007. "Characteristics of Vector Surge Relays for Distributed Synchronous Generator Protection.” Electric power systems research 77(2): 170-80.

Girgis, Adly A, Wenbin Chang, and Elham B Makram. 1990. "Analysis of HighImpedance Fault Generated Signals Using a Kalman Filtering Approach.” IEEE Transactions on Power delivery 5(4): 1714-24.

Grainger, John J, William D Stevenson, and William D Stevenson. 2003. Power System Analysis.

Grossmann, Alexander, and Jean Morlet. 1984. "Decomposition of Hardy Functions into Square Integrable Wavelets of Constant Shape." SIAM journal on mathematical analysis 15(4): 723-36.

\section{Hasheminejad, Saeid, Seyed Ghodratollah Seifossadat, Morteza Razaz, and Mahmood}

Joorabian. 2016. "Ultra-High-Speed Protection of Transmission Lines Using Traveling Wave Theory." Electric Power Systems Research 132: 94-103. 
He, Qian, and Rick S Blum. 2011. "New Hypothesis Testing-Based Methods for Fault Detection for Smart Grid Systems.” In 2011 45th Annual Conference on Information Sciences and Systems, IEEE, 1-6.

\section{Honorato, André Gustavo da Silva Melo, Gustavo Barbosa Lima da Silva, and Celso} Augusto Guimarães Santos. 2018. "Monthly Streamflow Forecasting Using NeuroWavelet Techniques and Input Analysis.” Hydrological Sciences Journal 63(15-16): $2060-75$.

Jiang, Zhongyuan, Zhiwu Li, Naiqi Wu, and Mengchu Zhou. 2017. “A Petri Net Approach to Fault Diagnosis and Restoration for Power Transmission Systems to Avoid the Output Interruption of Substations.” IEEE Systems Journal 12(3): 2566-76.

Kubat, Miroslav. 1999. "Neural Networks: A Comprehensive Foundation by Simon Haykin, Macmillan, 1994, ISBN 0-02-352781-7.” The Knowledge Engineering Review 13(4): 409-12.

Kumpulainen, L K, and K T Kauhaniemi. 2004. "Analysis of the Impact of Distributed Generation on Automatic Reclosing.” In IEEE PES Power Systems Conference and Exposition, 2004., IEEE, 603-8.

Nan, Cen, F Khan, and M T Iqbal. 2007. “Abnormal Process Condition Prediction (Fault Diagnosis) Using G2 Expert System.” In 2007 Canadian Conference on Electrical and Computer Engineering, IEEE, 1507-10.

Ndou, R, J I Fadiran, S Chowdhury, and S P Chowdhury. 2013. "Performance Comparison of Voltage and Frequency Based Loss of Grid Protection Schemes for Microgrids.” In 2013 IEEE Power \& Energy Society General Meeting, IEEE, 1-5.

Rawat, Sarvesh S S et al. 2014. “Anomaly Detection in Smart Grid Using Rough Set Theory and K Cross Validation.” In 2014 International Conference on Circuits, Power and Computing Technologies [ICCPCT-2014], IEEE, 479-83.

Reedy, R M, and W A Elsmore. 1983. "Electromechanical Relay to Detect Fallen 
Distribution Conductors." In APPA, Conf.,.

Samanta, B. 2004. “Gear Fault Detection Using Artificial Neural Networks and Support Vector Machines with Genetic Algorithms.” Mechanical systems and signal processing 18(3): $625-44$.

Samui, Ankita, and S R Samantaray. 2012. "Wavelet Singular Entropy-Based Islanding Detection in Distributed Generation." IEEE transactions on power delivery 28(1): 41118.

Veljko, M Todorovic, R Tadic Predrag, and M Djurovic Zeljko. 2010. "Expert System for Fault Detection and Isolation of Coal-Shortage in Thermal Power Plants.” In 2010 Conference on Control and Fault-Tolerant Systems (SysTol), IEEE, 666-71.

Wester, Craig G. 1998. "High Impedance Fault Detection on Distribution Systems.” In 1998 Rural Electric Power Conference Presented at 42nd Annual Conference, IEEE, c5-1.

Xin-min, Tao, Chen Wan-Hai, Du Bao-Xiang, and Dong Han-Guang. 2007. “A Novel Model of One-Class Bearing Fault Detection Using RNCS Algorithm Based on HOS.” In 2007 2nd IEEE Conference on Industrial Electronics and Applications, IEEE, 965-70.

Xu, X, and M Kezunovic. 2002. “Automated Feature Extraction from Power System Transients Using Wavelet Transform.” In Proceedings. International Conference on Power System Technology, IEEE, 1994-98.

Xu, Xiuping, and James F Peters. 2002. "Rough Set Methods in Power System Fault Classification.” In IEEE CCECE2002. Canadian Conference on Electrical and Computer Engineering. Conference Proceedings (Cat. No. O2CH37373), IEEE, 100105.

Yang, S K. 2003. “A Condition-Based Failure-Prediction and Processing-Scheme for Preventive Maintenance.” IEEE Transactions on Reliability 52(3): 373-83.

Yongli, Zhu, Huo Limin, and Lu Jinling. 2006. "Bayesian Networks-Based Approach for Power Systems Fault Diagnosis.” IEEE Transactions on Power Delivery 21(2): 634-39. 